\title{
Boutet de Monvel's calculus and groupoids I
}

\author{
Johannes Aastrup, Severino T. Melo, Bertrand Monthubert, and Elmar Schrohe
}

\begin{abstract}
Can Boutet de Monvel's algebra on a compact manifold with boundary be obtained as the algebra $\Psi^{0}(G)$ of pseudodifferential operators on some Lie groupoid $G$ ? If it could, the kernel $\mathcal{E}$ of the principal symbol homomorphism would be isomorphic to the groupoid $\mathrm{C}^{*}$-algebra $C^{*}(G)$. While the answer to the above question remains open, we exhibit in this paper a groupoid $G$ such that $C^{*}(G)$ possesses an ideal $\mathcal{I}$ isomorphic to $\mathscr{E}$. In fact, we prove first that $\mathscr{G} \simeq \Psi \otimes \mathcal{K}$ with the $\mathrm{C}^{*}$-algebra $\Psi$ generated by the zero order pseudodifferential operators on the boundary and the algebra $\mathcal{K}$ of compact operators. As both $\Psi \otimes \mathcal{K}$ and $\mathcal{I}$ are extensions of $C\left(S^{*} Y\right) \otimes \mathcal{K}$ by $\mathcal{K}\left(S^{*} Y\right.$ is the co-sphere bundle over the boundary) we infer from a theorem by Voiculescu that both are isomorphic.
\end{abstract}

Mathematics Subject Classification (2010). 58J32, 19K56, 58H05, 58J40.

Keywords. Boundary value problems on manifolds, index theory, groupoids, KK-theory, extension theory.

\section{Introduction}

Boutet de Monvel's calculus [3], [4], [5], [11], [25], [29] is a pseudodifferential calculus on manifolds with boundary. It includes the classical differential boundary value problems as well as the parametrices to elliptic elements. Many operatoralgebraic aspects of this algebra (spectral invariance, noncommutative residues and traces, composition sequence, K-theory) have been studied recently [1], [10], [12], [19], [20], [23], [28]. The problem of identifying this algebra as the pseudodifferential algebra (or as an ideal of one) of a Lie groupoid may be the key to an effective application of the methods of noncommutative geometry. If that is accomplished, one could then seek for extensions of the calculus, and for a better understanding of its index theory [5], [9], [25]. Basic definitions and certain facts about Boutet de Monvel's algebra are recalled in Section 1.

The groupoid approach to pseudodifferential calculus was developed in noncommutative geometry, following the seminal work of A. Connes for foliations [8]. The guiding principle in that approach is that the central object in global analysis is a groupoid. To study a particular situation, for a class of singular manifolds for instance, one needs to define a groupoid adapted to the context and then use the general 
pseudodifferential tools for groupoids, as developed in [22], [24], [21], [17], [18]. This has been applied to the context of manifolds with corners, with the goal of studying Melrose's $b$-calculus (see [22], [24], [21]). Groupoids were defined whose pseudodifferential calculi recover the $b$-calculus and the cusp-calculus. Basic definitions and certain facts about pseudodifferential calculus on groupoids are recalled in Section 3.

The starting point of this work is the following result (see [19]): The kernel of the principal symbol map for Boutet de Monvel's calculus is equal to the norm closure $\mathcal{E}$ of the ideal of singular Green operators. Since in the pseudodifferential calculus on a groupoid, the $\mathrm{C}^{*}$-algebra of the groupoid is the kernel of the principal symbol map, this gives a hint that finding a groupoid whose $\mathrm{C}^{*}$-algebra is $\mathcal{E}$ could give some insight about the relationship between the Boutet de Monvel algebra and groupoid pseudodifferential algebras.

Besides, $\mathscr{E}$ fits into a short exact sequence (see [20], Section 7)

$$
0 \rightarrow \mathcal{K} \rightarrow \mathcal{E} \rightarrow C\left(S^{*} Y\right) \otimes \mathcal{K} \rightarrow 0,
$$

which is similar to that for pseudodifferential operators on smooth manifolds:

$$
0 \rightarrow \mathcal{K} \rightarrow \Psi \rightarrow C\left(S^{*} Y\right) \rightarrow 0 .
$$

In Section 2, we show that $\mathscr{Z}$ is actually Morita-equivalent to the norm closure $\Psi$ of the algebra of pseudodifferential operators on the boundary. Since $\mathcal{G}$ is a stable $\mathrm{C}^{*}$-algebra, it is thus isomorphic to $\Psi \otimes \mathcal{K}$.

On the other hand, we define in Section 4 a groupoid whose $C^{*}$-algebra contains an ideal $\mathcal{I}$ which fits in an extension analoguous as that of $\Psi \otimes \mathcal{K}$. By showing in Section 5 that the KK-theory elements induced by these extensions coincide, we infer from a theorem by Voiculescu that $\mathscr{E}$ and $\mathcal{I}$ are isomorphic.

Acknowledgements. J. Aastrup and E. Schrohe gratefully acknowledge the support of Deutsche Forschungsgemeinschaft through the project 'Deformation Theory for Boundary Value Problems' (Schr319/7-2).

S. T. Melo and B. Monthubert were partly funded by a cooperation agreement CAPES-COFECUB.

\section{Boutet de Monvel's calculus}

Let $X$ denote a compact manifold of dimension $n$ with boundary $Y$ and interior $\dot{X}$. Given a pseudodifferential operator $P$, defined on an open neighborhood $\widetilde{X}$ of $X$, and $u \in C^{\infty}(X)$, one defines $P_{+} u$ as equal to the restriction to $\dot{X}$ of $P E u$, where $E u$ is the extension of $u$ to $\tilde{X}$ which vanishes outside $X$. In general, singularities may develop at the boundary, and one gets only a mapping $P_{+}: C^{\infty}(X) \rightarrow C^{\infty}(\dot{X})$. One says that $P$ has the transmission property if the image of the truncated operator $P_{+}$is 
contained in $C^{\infty}(X)$ (a subspace of $C^{\infty}(\dot{X})$ ). This was defined by Boutet de Monvel in [3], [4], where he proved that the transmission property for a classical (polyhomogoneous) pseudodifferential operator is equivalent to certain symmetry conditions for the homogeneous components of the asymptotic expansion of its symbol at the boundary. Later [5], he constructed an algebra whose elements are operators of the form

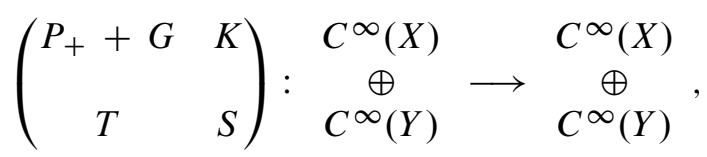

where $P$ is a pseudodifferential operator on $X$ satisfying a condition that ensures the transmission property, $S$ is a pseudodifferential operator on $Y$, while $G, K$ and $T$ belong to classes of operators he then defined and named, respectively, singular Green, Poisson and trace operators. We call an operator as in (1) a Boutet de Monvel operator. For detailed expositions of his calculus, we refer to [11], [25].

A Boutet de Monvel operator has an order, roughly the usual order of pseudodifferential operators. The entries $T$ and $G$ have, moreover, an integer class assigned to them. The class of a trace operator is related to the order of the derivatives that appear in the boundary-value conditions it prescribes. One must assign a class also to singular Green operators due to the fact that the composition $K T$ is a singular Green operator. There exist isomorphisms between suitable Sobolev spaces such that the composition of a given operator of arbitrary order and class with one of them has order and class zero. For index theory purposes it is therefore sufficient to consider operators of order and class zero. These form an adjoint invariant subalgebra of the algebra $\mathscr{L}(\mathscr{H})$ of all bounded operators on a suitable Hilbert space $\mathscr{H}$. Adopting the definition of order in [25], [29] for $K$ and $T$, we here choose $\mathscr{H}=L^{2}(X) \oplus L^{2}(Y)$. If, as does Grubb [11], one keeps the original definition (which makes more sense if one needs general $L^{p}$ estimates) then one must take a Sobolev space of order $-1 / 2$ over the boundary.

Boutet de Monvel operators can also be defined as mappings between smooth sections of vector bundles. If $E$ is a bundle of positive rank over $X$, and $F$ is an arbitrary bundle over $Y$, then the algebra of all Boutet de Monvel operators of order and class zero acting between sections of $E$ and $F$ is Morita equivalent [19], Section 1.5, to the algebra obtained by taking a rank-one trivial bundle over $X$ and the zero-bundle over $Y$. This partly justifies, again if one is interested in index theory, to consider only the operators appearing in the upper-left corner of the matrix in (1) and to assume, as we did at the beginning, that the bundle over $X$ is $X \times \mathbb{C}$.

The problem of computing the Fredholm index of a Boutet de Monvel operator acting between sections of different bundles over each side can be reduced to the case of equal bundles on both sides by a device developed by Boutet de Monvel [3], [4], recalled in [20], Section 1.1.

Let us now explain what a singular Green operator $G$ is, in the case of order and class zero and of a rank-one trivial bundle over $X$. Its distribution kernel is smooth outside the boundary diagonal; i.e., if $\varphi \in C_{c}^{\infty}(\dot{X})$, and if we denote by $M_{\varphi}$ the 
operator of multiplication by $\varphi$, then $G M_{\varphi}$ and $M_{\varphi} G$ are integral operators with smooth kernels. The push-forward of $G$ by a boundary chart is an operator-valuedsymbol pseudodifferential operator on the variables tangential to the boundary, as we describe below. It is perhaps worth stressing, however, that it is in general not a pseudodifferential operator on all variables, because of its particular way of acting on the normal variable.

Given $u \in C_{c}^{\infty}\left(\mathbb{R}_{+}^{n}\right), \mathbb{R}_{+}^{n}=\left\{\left(x^{\prime}, x_{n}\right) \in \mathbb{R}^{n-1} \times \mathbb{R} ; x_{n} \geq 0\right\}$, let $\hat{u}$ denote the vector-valued Fourier transform of $u$ with respect to the $n-1$ first variables,

$$
\hat{u}\left(\xi^{\prime}\right)=\int e^{i x^{\prime} \cdot \xi^{\prime}} u\left(x^{\prime}, \cdot\right) d x^{\prime} \in C_{c}^{\infty}\left(\mathbb{R}_{+}\right) .
$$

In local coordinates for which the boundary corresponds to $x_{n}=0$ and the interior to $x_{n}>0, G$ is given by

$$
G u\left(x^{\prime}, \cdot\right)=(2 \pi)^{1-n} \int e^{i x^{\prime} \cdot \xi^{\prime}} g\left(x^{\prime}, \xi^{\prime}, D_{n}\right) \hat{u}\left(\xi^{\prime}\right) d \xi^{\prime}
$$

The integrals in (2) or in (3) should be regarded, for fixed $\xi^{\prime}$ or $x^{\prime}$, respectively, as $L^{2}\left(\mathbb{R}_{+}\right)$-valued integrals. For each $\left(x^{\prime}, \xi^{\prime}\right), g\left(x^{\prime}, \xi^{\prime}, D_{n}\right)$ in (3) is an integral operator with kernel $\tilde{g}\left(x^{\prime}, \cdot, \cdot, \xi^{\prime}\right)$ equal to the restriction to $\mathbb{R}_{+} \times \mathbb{R}_{+}$of a function belonging to the Schwartz space of rapidly decreasing functions on $\mathbb{R}^{2}$. The function $\tilde{g}\left(x^{\prime}, x_{n}, y_{n}, \xi^{\prime}\right)$ (called by Grubb the symbol-kernel of $\left.G\right)$ is smooth and satisfies the estimates [11], (1.2.38). This is invariantly defined [11], Theorem 2.4.11, with respect to coordinate changes that preserve the set $\left\{x_{n}=0\right\}$.

We denote by $\mathcal{A}_{0}$ the set of all polyhomogeneous operators $P_{+}+G$ of order and class zero on $X$, and by $\mathscr{E}_{0}$ its subset of all singular Green operators. It follows from the rules of Boutet de Monvel's calculus that $\mathcal{A}_{0}$ is an algebra and that $\boldsymbol{E}_{0}$ is an ideal in $\mathcal{A}_{0}$.

In the sequel, we shall restrict ourselves to coordinate changes which preserve the variable $x_{n}$, i.e., we choose a normal coordinate. Then two *-homomorphisms are defined on $\mathcal{A}_{0}$, the principal symbol and the boundary principal symbol:

$$
\sigma: \mathcal{A}_{0} \rightarrow C^{\infty}\left(S^{*} X\right) \text { and } \gamma: \mathcal{A}_{0} \rightarrow C^{\infty}\left(S^{*} Y, \mathscr{L}\left(L^{2}\left(\mathbb{R}_{+}\right)\right)\right) .
$$

The principal symbol of a given $P_{+}+G$ is, by definition, the usual principal symbol of $P$

$$
\sigma\left(P_{+}+G\right)=p_{0}
$$

where $p_{0}$ is the leading term in the asymptotic expansion of the symbol of $P$.

At a point $\left(x^{\prime}, \xi^{\prime}\right)$ in $S^{*} Y$, the boundary principal symbol of $P_{+}$is defined to be the truncated Fourier multiplier

$$
\gamma_{P_{+}}\left(x^{\prime}, \xi^{\prime}\right)=p_{0}\left(x^{\prime}, 0, \xi^{\prime}, D_{n}\right)_{+}
$$

of symbol $\xi_{n} \mapsto p_{0}\left(x^{\prime}, 0, \xi^{\prime}, \xi_{n}\right)$. The boundary principal symbol of $G \in \mathscr{G}_{0}$ is the integral operator

$$
\gamma_{G}\left(x^{\prime}, \xi^{\prime}\right)=g_{0}\left(x^{\prime}, \xi^{\prime}, D_{n}\right)
$$


with the rapidly decreasing kernel $\tilde{g}_{0}\left(x^{\prime}, \cdot, \cdot, \xi^{\prime}\right)$, where $\tilde{g}_{0}$ denotes the leading term in the asymptotic expansion of $\tilde{g}$, cf. [11], (1.2.39). Then $\gamma$ maps $\mathscr{E}_{0}$ into $C^{\infty}\left(S^{*} Y, \mathcal{K}_{\mathbb{R}_{+}}\right)$, with the ideal $\mathcal{K}_{\mathbb{R}_{+}}$of compact operators on $L^{2}\left(\mathbb{R}_{+}\right)$.

Let $\mathcal{A}$ and $\mathscr{G}$ denote the norm closures of $\mathcal{A}_{0}$ and $\mathscr{E}_{0}$, respectively; and let $\mathcal{K}_{X}$ denote the set of all compact operators on $L^{2}(X)$. It follows from Theorem 1 in [25], 2.3.4.4, that $\sigma$ and $\gamma$ can be extended to $\mathrm{C}^{*}$-algebra homomorphisms

$$
\bar{\sigma}: \mathcal{A} \rightarrow C\left(S^{*} X\right) \text { and } \bar{\gamma}: \mathcal{A} \rightarrow C\left(S^{*} Y, \mathscr{L}\left(L^{2}\left(\mathbb{R}_{+}\right)\right)\right) .
$$

Moreover, by Corollary 2 in [25], 2.3.4.4, and [19], Theorems 5 and 6, we have that

$$
\operatorname{ker} \bar{\gamma} \cap \operatorname{ker} \bar{\sigma}=\mathcal{K}_{X}, \quad \operatorname{ker} \bar{\sigma}=\mathscr{G},
$$

and $\bar{\gamma}$ maps $\mathcal{G}$ onto $C\left(S^{*} Y, \mathcal{K}_{\mathbb{R}_{+}}\right)$. In other words, the restriction of the boundary principal symbol to $\mathscr{G}$ gives rise to the exact sequence of $\mathrm{C}^{*}$-algebras

$$
0 \rightarrow \mathcal{K}_{X} \rightarrow \mathscr{\mathcal { Y }} \stackrel{\bar{\gamma}}{\rightarrow} C\left(S^{*} Y, \mathcal{K}_{\mathbb{R}_{+}}\right) \rightarrow 0 .
$$

In Section 2 we use (6) to prove that $\mathscr{Y}$ is isomorphic to the tensor product $\Psi \otimes \mathcal{K}$ of the $\mathrm{C}^{*}$-closure $\Psi$ of the pseudodifferential operators of order zero on $Y$ by the compacts. For that we need to use trace and Poisson operators.

Similarly as for the singular Green operators, the trace operators and the Poisson operators ( $T$ and $K$ in (1)) are, locally, operator-valued-symbol pseudodifferential operators on the variables tangential to the boundary, given by

$$
T u\left(x^{\prime}\right)=(2 \pi)^{1-n} \int e^{i x^{\prime} \cdot \xi^{\prime}} t\left(x^{\prime}, \xi^{\prime}, D_{n}\right) \hat{u}\left(\xi^{\prime}\right) d \xi^{\prime}, \quad u \in C_{c}^{\infty}\left(\mathbb{R}_{+}^{n}\right),
$$

and

$$
K u\left(x^{\prime}, \cdot\right)=(2 \pi)^{1-n} \int e^{i x^{\prime} \cdot \xi^{\prime}} k\left(x^{\prime}, \xi^{\prime}, D_{n}\right) \hat{u}\left(\xi^{\prime}\right) d \xi^{\prime}, \quad u \in C_{c}^{\infty}\left(\mathbb{R}^{n-1}\right) .
$$

The mappings $t\left(x^{\prime}, \xi^{\prime}, D_{n}\right): L^{2}\left(\mathbb{R}_{+}\right) \rightarrow \mathbb{C}$ and $k\left(x^{\prime}, \xi^{\prime}, D_{n}\right): \mathbb{C} \rightarrow L^{2}\left(\mathbb{R}_{+}\right)$are defined, for each $\left(x^{\prime}, \xi^{\prime}\right) \in \mathbb{R}^{n-1} \times \mathbb{R}^{n-1}$, each $v \in L^{2}\left(\mathbb{R}_{+}\right)$and each $\alpha \in \mathbb{C}$, by

$$
t\left(x^{\prime}, \xi^{\prime}, D_{n}\right) v=\int \tilde{t}\left(x^{\prime}, y_{n}, \xi^{\prime}\right) v\left(y_{n}\right) d y_{n}
$$

and

$$
\left[k\left(x^{\prime}, \xi^{\prime}, D_{n}\right) \alpha\right]\left(x_{n}\right)=\alpha \tilde{k}\left(x^{\prime}, x_{n}, \xi^{\prime}\right) .
$$

For each $\left(x^{\prime}, \xi^{\prime}\right), \tilde{t}\left(x^{\prime}, \cdot, \xi^{\prime}\right)$ and $\tilde{k}\left(x^{\prime}, \cdot, \xi^{\prime}\right)$ are restrictions to $\mathbb{R}_{+}$of functions in the Schwartz class on $\mathbb{R}$. The functions $\tilde{t}\left(x^{\prime}, y_{n}, \xi^{\prime}\right)$ and $\tilde{k}\left(x^{\prime}, x_{n}, \xi^{\prime}\right)$, called the symbolkernels of $T$ and $K$, are smooth and satisfy certain estimates. In the polyhomogenous case, they have asymptotic expansions in homogeneous components, whose leading terms we denote by $\tilde{t}_{0}$ and $\tilde{k}_{0}$, respectively. The estimates and expansions for $\tilde{t}$ and $\tilde{k}$ 
listed or explained in [11], Section 1.2, are not the right ones for our definition of order (and consequent choice of Hilbert space): we need to shift some of the exponents there by $\pm 1 / 2$.

The boundary-principal symbols of $T$ and $K$ are

$$
\gamma_{T}\left(x^{\prime}, \xi^{\prime}\right)=t_{0}\left(x^{\prime}, \xi^{\prime}, D_{n}\right) \text { and } \gamma_{K}\left(x^{\prime}, \xi^{\prime}\right)=k_{0}\left(x^{\prime}, \xi^{\prime}, D_{n}\right),
$$

defined as in (9) and (10), except that $\tilde{t}_{0}$ and $\tilde{k}_{0}$ replace $\tilde{t}$ and $\tilde{k}$. Lastly, the boundary principal symbol of a polyhomogeneous pseudodifferential operator on $Y$ is simply its usual principal symbol, and we get a *-homomorphism

$$
\gamma: \mathbb{B}_{0} \rightarrow C^{\infty}\left(S^{*} Y, \mathscr{L}\left(L^{2}\left(\mathbb{R}_{+}\right) \oplus \mathbb{C}\right)\right),
$$

where $\mathscr{B}_{0}$ denotes the set of all polyhomogeneous Boutet de Monvel operators of order and class zero on $X$. It has a continuous extension to the norm closure of $\mathcal{B}_{0}$, but we will not use this fact.

\section{A product description of the singular Green operators}

Lemma 1. There exists a zero-order Poisson operator $K$ such that $K^{*} K$ is a strictly positive operator on $L^{2}(Y)$.

Proof. It is well known that the Dirichlet problem

$$
\left(\begin{array}{c}
\Delta \\
\gamma_{0}
\end{array}\right): H^{2}(X) \longrightarrow \underset{H^{3 / 2}(Y)}{\bigoplus}
$$

defines a bounded invertible operator. We denote by $\lambda^{3 / 2}$ an order reduction of order $3 / 2$ on $Y$ and by $\Lambda^{-2}$ an order reduction of order -2 on $X$. This gives us an isomorphism

$$
\left(\begin{array}{c}
\Delta \Lambda^{-2} \\
\lambda^{3 / 2} \gamma_{0} \Lambda^{-2}
\end{array}\right)=\left(\begin{array}{cc}
1 & 0 \\
0 & \lambda^{3 / 2}
\end{array}\right)\left(\begin{array}{c}
\Delta \\
\gamma_{0}
\end{array}\right) \Lambda^{-2}: L^{2}(X) \longrightarrow \begin{gathered}
L^{2}(X) \\
L^{2}(Y)
\end{gathered}
$$

which is an element of order and class 0 in Boutet de Monvel's calculus. Its inverse therefore also is in Boutet de Monvel's calculus; it is of the form

$$
\left(P_{+}+G K\right): \underset{L^{2}(Y)}{\bigoplus} \longrightarrow L^{2}(X)
$$

with suitable $P, G$, and $K$ of order and class zero. In particular, $K$ is a right inverse for the trace operator $T=\lambda^{3 / 2} \gamma_{0} \Lambda^{-2}: L^{2}(X) \rightarrow L^{2}(Y)$. For $v \in L^{2}(Y)$ we thus have

$$
\|v\|_{L^{2}(Y)}=\|T K v\|_{L^{2}(Y)} \leq\|T\|_{\mathscr{L}\left(L^{2}(X), L^{2}(Y)\right)}\|K v\|_{L^{2}(X)} .
$$

We then get $\|K v\| \geq c\|v\|$ for some $c>0$, so that $K^{*} K$ is strictly positive. 
Lemma 2. There exist a trace operator of order and class zero $T: L^{2}(X) \rightarrow L^{2}(Y)$ and a Poisson operator of order zero $K: L^{2}(Y) \rightarrow L^{2}(X)$ such that $T K$ is equal to the identity operator on $L^{2}(Y), K^{*}=T$ and $T^{*}=K$.

Proof. Let $K_{0}$ be a zero-order Poisson operator such that $K_{0}^{*} K_{0}$ is a strictly positive operator on $L^{2}(Y)$, and let $Q=\left(K_{0}^{*} K_{0}\right)^{-1 / 2} . Q$ is a zero-order pseudodifferential operator on $Y$. Take $K=K_{0} Q$ and $T=Q K_{0}^{*}$.

We denote by $\Psi$ the norm closure of the algebra of all polyhomogeneous pseudodifferential operators of order zero on $Y$, and by $\bar{\sigma}: \Psi \rightarrow C\left(S^{*} Y\right)$ the continuous extension of the principal-symbol homomorphism. It is well known (this is mentioned in [2] and follows from [15], Theorem A.4, or from [13], Theorem 3.3) that $\bar{\sigma}$ induces the short exact sequence of $\mathrm{C}^{*}$-algebras

$$
0 \longrightarrow \mathcal{K}_{Y} \longrightarrow \Psi \stackrel{\bar{\sigma}}{\longrightarrow} C\left(S^{*} Y\right) \longrightarrow 0,
$$

where $\mathcal{K}_{Y}$ denotes the ideal of compact operators on $L^{2}(Y)$.

By Lemma 2, a $C^{*}$-homomorphism $\Xi: \Psi \rightarrow \mathscr{E}$ can be defined by

$$
\Xi(A)=K A T \text {. }
$$

Since $\Xi(A)$ is compact if $A$ is compact, we can use $\Xi$ to couple the sequences (6) and (11). Together they yield the commutative diagram of exact sequences of $C^{*}$-algebras

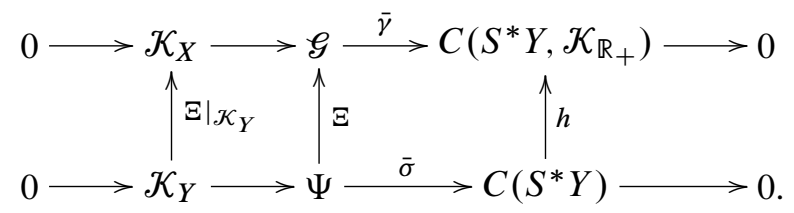

Lemma 3. The homomorphism $\Xi$ imbeds $\Psi$ as a hereditary subalgebra of $\mathscr{E}$.

Proof. We have to prove that, if $0 \leq G \leq K A T$ then $G$ is again of the form $K A_{1} T$ with $A_{1} \in \Psi$. Since $K T$ acts as the identity on $K A T$ it also acts as the identity on $G$ and we therefore get $G=K T G K T=K(T G K) T$.

Lemma 4. Let

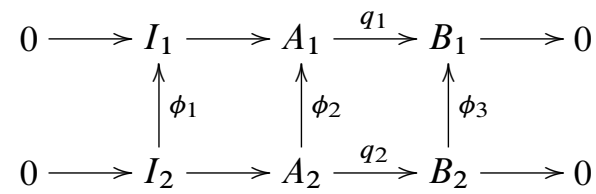

be a commutative diagram of short exact sequences, where $\phi_{1}, \phi_{2}$ and $\phi_{3}$ are embeddings. Then $\phi_{2}$ is full provided that $\phi_{1}$ and $\phi_{3}$ are full. 
Proof. We have to prove that the two-sided ideal generated by $\phi_{2}\left(A_{2}\right)$ is dense in $A_{1}$. We thus have to prove that to a given $a \in A_{1}$ and a given $\varepsilon>0$ we can find an element $b$ in the two-sided ideal generated by the image of $\phi_{2}$ such that $\|a-b\|<\varepsilon$. Since $\phi_{3}$ is full, we can find an element $c$ in the two-sided ideal generated by $\phi_{3}\left(B_{2}\right)$ such that $\left\|q_{1}(a)-c\right\|<\frac{\varepsilon}{2}$. The element $c$ can be lifted to an element $b_{1}$ in the two-sided ideal generated by $\phi_{2}\left(A_{2}\right)$ and we can therefore find an element $d_{1} \in I_{1}$ such that $\left\|a-b_{1}-d_{1}\right\|<\frac{\varepsilon}{2}$. Since $\phi_{1}$ is full, there is an element $d_{2}$ in the two-sided ideal generated by $\phi_{1}\left(I_{2}\right)$ with $\left\|d_{1}-d_{2}\right\|<\frac{\varepsilon}{2}$. As the desired $b$ we can therefore choose $b=b_{1}+d_{2}$.

Theorem 1. The algebras $\mathcal{E}$ and $\Psi \otimes \mathcal{K}$ are isomorphic.

Proof. By Lemma 3, the diagram (12) and Lemma 4, the imbedding $\Xi$ is full and hereditary. It follows from the remark below Theorem 8 on p. 155 in [8] that $\mathcal{E}$ and $\Psi$ are strongly Morita equivalent. By the results in [6] and [7], $\mathscr{E} \otimes \mathcal{K}$ is isomorphic to $\Psi \otimes \mathcal{K}$. However $\mathcal{E}$ is stable, since it is the extension of $\mathcal{K}$ with a stable algebra, namely $C\left(S^{*} Y, \mathcal{K}_{\mathbb{R}_{+}}\right)$, see Proposition 6.12 in [27]). This gives the isomorphism.

\section{Pseudodifferential operators and groupoids}

Groupoids were introduced in the context of global analysis when A. Connes showed that in the case of foliations the index takes values in a $\mathrm{C}^{*}$-algebra which is defined as the $\mathrm{C}^{*}$-algebra of the holonomy groupoid of the foliation. He defined a pseudodifferential calculus on a foliation using the groupoid structure.

In several papers ([22], [24], [17], [21]), generalizations of this approach to a larger class of groupoids were achieved. One particular aspect of this theory is that, as A. Connes showed in [8] for smooth manifolds, it is possible to define the analytic index using a groupoid, the tangent groupoid.

A groupoid is a small category in which all morphisms are invertible. This means that a groupoid $G$ has a set of units, denoted by $G^{(0)}$, and two maps called range and source, $G \stackrel{r}{\stackrel{s}{\longrightarrow}} G^{(0)}$.

Two elements $\gamma, \gamma^{\prime} \in G$ are composable if and only if $r(\gamma)=s\left(\gamma^{\prime}\right)$ :

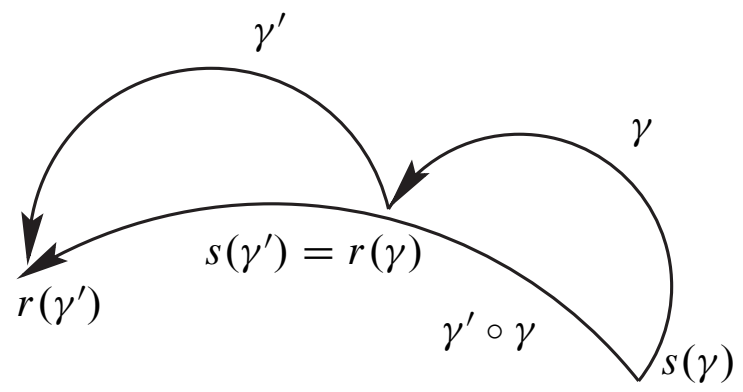


We recall briefly the main aspects of this theory. Let $G$ be a Lie groupoid, which means that it has a smooth structure. Then one can define an algebra of pseudodifferential operators $\Psi^{\infty}(G)$ : A pseudodifferential operator on $G$ is a $G$ equivariant continuous family of pseudodifferential operators on the fibers of $G$.

For example, if $M$ is a manifold without boundary, and $G=M \times M$, with set of units $G^{(0)}=M$, and range and source maps $r(x, y)=x, s(x, y)=y$, and composition $(x, y)(y, z)=(x, z)$, then $\Psi^{\infty}(G)$ is the algebra of pseudodifferential operators on $M$.

If $G$ is a Lie group, $\Psi^{\infty}(G)$ is the algebra of $G$-equivariant pseudodifferential operators on $G$.

In order to work with singular manifolds, the framework of Lie groupoids needs to be extended. That was done in [17], where the algebras of pseudodifferential operators on continuous family groupoids, which are groupoids whose fibers are smooth manifolds, were defined.

On the algebra of pseudodifferential operators one can define a symbol map, $\sigma$. The algebra of order 0 operators can be completed as a $\mathrm{C}^{*}$-algebra, denoted by $\overline{\Psi^{0}}(G)$, and the symbol map extends to this algebra. The "regularizing operators" of the calculus, which are the operators with trivial symbol, are the elements of the $\mathrm{C}^{*}$-algebra of the groupoid, and we have the Atiyah-Singer exact sequence

$$
0 \rightarrow C^{*}(G) \rightarrow \overline{\Psi^{0}}(G) \rightarrow C\left(S^{*}(G)\right) \rightarrow 0,
$$

where $S^{*}(G)$ is the cosphere bundle of the Lie algebroid $A(G)$, which can be thought of as a tangent space.

We next recall in more detail the construction of the adiabatic groupoid ad $(Y \times Y)$ associated with a smooth manifold $Y$ :

$$
{ }^{\mathrm{ad}}(Y \times Y)=(T Y \times\{0\}) \cup\left(Y \times Y \times \mathbb{R}_{+}^{*}\right)
$$

with the tangent bundle $T Y$ of $Y$. The groupoid structure is given as follows:

$$
\begin{gathered}
r(x, \xi, 0)=s(x, \xi, 0)=x,(x, \xi, 0) \circ\left(x, \xi^{\prime}, 0\right)=\left(x, \xi+\xi^{\prime}, 0\right), \\
r(x, y, \lambda)=(x, \lambda), s(x, y, \lambda)=(y, \lambda),(x, y, \lambda) \circ(y, z, \lambda)=(x, z, \lambda), \quad \lambda>0 .
\end{gathered}
$$

This groupoid is endowed with a differential structure, through an exponential, in the following way:

- On $Y \times Y \times \mathbb{R}_{+}^{*}$, the structure is that of a product of manifolds.

- Define a map on an open neighborhood $U$ of $T Y \times\{0\}$ in $T Y \times \mathbb{R}_{+}$, with values in $^{\text {ad }}(Y \times Y)$, by

$$
\left\{\begin{array}{l}
\psi(x, \xi, \lambda)=\left(x, \exp _{x}(-\lambda \xi), \lambda\right) \quad \text { if } \lambda>0 \\
\psi(x, \xi, 0)=(x, \xi, 0)
\end{array}\right.
$$


In other terms, the topology is such that a sequence of terms $\left(x_{n}, y_{n}, \lambda_{n}\right)$ of $Y \times Y \times \mathbb{R}_{+}^{*}$ converges to $(x, \xi, 0) \in T Y \times\{0\}$, if and only if we have locally

$$
x_{n} \rightarrow x, \quad y_{n} \rightarrow x, \quad \frac{x_{n}-y_{n}}{\lambda_{n}} \rightarrow \xi .
$$

Note that A. Connes' tangent groupoid is just the restriction of ${ }^{\text {ad }}(Y \times Y)$ to $\lambda \in[0,1]$.

The main interest of this groupoid is that it provides a way to define the analytic index. Consider indeed the decomposition of the groupoid as an open and a closed subgroupoid, which gives rise to the exact sequence

$$
0 \rightarrow C^{*}\left(Y \times Y \times \mathbb{R}_{+}^{*}\right) \rightarrow C^{*}\left({ }^{\mathrm{ad}}(Y \times Y)\right) \rightarrow C^{*}(T Y) \rightarrow 0 .
$$

This simplifies since $C^{*}(Y \times Y) \simeq \mathcal{K}$ and $C^{*}(T Y) \simeq C_{0}\left(T^{*} Y\right)$. A. Connes proved that the boundary map of the 6-terms exact sequence induced by this extension is nothing but the analytic index

$$
\operatorname{ind}_{a}: K_{c}^{0}\left(T^{*} Y\right) \rightarrow K_{1}\left(C_{0}(\mathbb{R}) \otimes \mathcal{K}\right)=\mathbb{Z} .
$$

\section{A groupoid associated to the singular Green operators}

Suppose that we could identify the $\mathrm{C}^{*}$-closure of Boutet de Monvel's algebra with the $C^{*}$-algebra $\Psi^{0}(G)$ of pseudodifferential operators on a Lie groupoid $G$. Then, as pointed out above, the kernel of the principal symbol map would be isomorphic to $C^{*}(G)$. As the kernel of the principal symbol map in Boutet de Monvel's calculus consists of the singular Green operators, we thus wish to identify these with the $\mathrm{C}^{*}$-algebra of a groupoid.

We will actually not identify them with a groupoid $C^{*}$-algebra but with an ideal in a groupoid $\mathrm{C}^{*}$-algebra.

Let us consider the following action of the group $\mathbb{R}_{+}^{*}$ on ${ }^{\text {ad }}(Y \times Y)$ :

- On $T Y, \mathbb{R}_{+}^{*}$ acts by dilations: $\lambda \cdot(x, \xi)=(x, \lambda \xi)$.

- On $Y \times Y \times \mathbb{R}_{+}^{*}, \mathbb{R}_{+}^{*}$ acts by $\lambda \cdot(x, y, t)=\left(x, y, \frac{t}{\lambda}\right)$.

This is a continuous action: If $\left(x_{n}, y_{n}, t_{n}\right)$ converges to $(x, \xi)$ (which means that $\left.x_{n}, y_{n} \rightarrow x, t_{n} \rightarrow 0, \frac{x_{n}-y_{n}}{t_{n}} \rightarrow \xi\right)$, then $\lambda \cdot\left(x_{n}, y_{n}, t_{n}\right)=\left(x_{n}, y_{n}, \frac{t_{n}}{\lambda}\right) \rightarrow(x, \lambda \xi, 0)$ since

$$
\frac{x_{n}-y_{n}}{\frac{t_{n}}{\lambda}} \rightarrow \lambda \xi \text {. }
$$

It is thus possible to construct the semi-direct product $G={ }^{\text {ad }}(Y \times Y) \rtimes \mathbb{R}_{+}^{*}$ of the adiabatic groupoid by $\mathbb{R}_{+}:$As a set it is ${ }^{\text {ad }}(Y \times Y) \times \mathbb{R}_{+}^{*}$, with set of units $Y \times \mathbb{R}_{+}$, such that

$$
\text { - } r(x, y, t, \lambda)=(x, t), s(x, y, t, \lambda)=\left(y, \frac{t}{\lambda}\right) \text { for } t>0 \text {; }
$$


- $r(x, \xi, \lambda)=(x, 0), s(x, \xi, \lambda)=(x, 0)$ for $t=0$;

- $(x, y, t, \lambda)\left(y, z, \frac{t}{\lambda}, \mu\right)=(x, z, t, \lambda \mu)$;

- $(x, \xi, \lambda)(x, \eta, \mu)=(x, \xi+\lambda \eta, \lambda \mu))$.

Note that the action of $\mathbb{R}_{+}^{*}$ on the adiabatic groupoid induces an action on its $\mathrm{C}^{*_{-}}$ algebra, and that J. Renault proved in [26] that for any locally compact groupoid $\mathscr{E}$ one has

$$
C^{*}\left(\mathscr{G} \rtimes \mathbb{R}_{+}^{*}\right) \simeq C^{*}(\mathscr{G}) \rtimes \mathbb{R}_{+}^{*} .
$$

The evaluation at $t=0$ provides a map $e_{0}: C^{*}(G) \rightarrow C_{0}\left(T^{*} Y\right) \rtimes \mathbb{R}_{+}^{*}$. Also, the evaluation at the zero-section $\xi=0$ induces a map $r_{0}: C_{0}\left(T^{*} Y\right) \rtimes \mathbb{R}_{+}^{*} \rightarrow C(Y) \rtimes \mathbb{R}_{+}^{*}$.

But since the action of $\mathbb{R}_{+}^{*}$ on $Y$ is trivial, the latter algebra is just the algebra of the (regular) product:

$$
C(Y) \rtimes \mathbb{R}_{+}^{*}=C_{0}\left(Y \times \mathbb{R}_{+}^{*}\right) .
$$

Let $C=\operatorname{ker} r_{0}$ and $\mathcal{I}=\operatorname{ker} r_{0} \circ e_{0}$.

The kernel of $e_{0}$ is $C^{*}\left(Y \times Y \times \mathbb{R}_{+}^{*} \rtimes \mathbb{R}_{+}^{*}\right)$. But $\mathbb{R}_{+}^{*} \rtimes \mathbb{R}_{+}^{*}$ is directly isomorphic to the pair groupoid $\mathbb{R}_{+}^{*} \times \mathbb{R}_{+}^{*}$ : To clarify the proof, let us denote $G_{1}=\mathbb{R}_{+}^{*} \rtimes \mathbb{R}_{+}^{*}$ and $G_{2}=\mathbb{R}_{+}^{*} \times \mathbb{R}_{+}^{*}$. Then let $\phi: G_{1} \rightarrow G_{2}$ be defined by

$$
\phi(t, \lambda)=\left(t, \frac{t}{\lambda}\right) .
$$

This a morphism of groupoids: The composition of $(t, \lambda)$ with $\left(\frac{t}{\lambda}, \mu\right)$ gives $(t, \lambda \mu)$, and

while

$$
\phi(t, \lambda \mu)=\left(t, \frac{t}{\lambda \mu}\right),
$$

$$
\phi(t, \lambda) \circ \phi\left(\frac{t}{\lambda}, \mu\right)=\left(t, \frac{t}{\lambda}\right) \circ\left(\frac{t}{\lambda}, \frac{t}{\lambda \mu}\right)=\left(t, \frac{t}{\lambda \mu}\right) .
$$

Hence the kernel of $e_{0}$ is just the algebra of compact operators, $\mathcal{K}$.

To make this clear, here is the commutative diagram describing this:

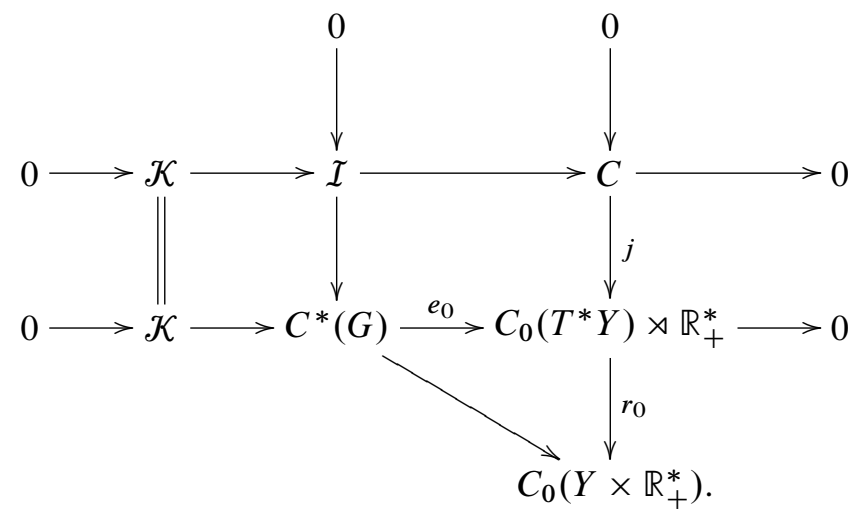


We will use this diagram and extension theory to prove that $\mathcal{I}$ is isomorphic to the algebra of singular Green operators.

Proposition 1. $C$ is isomorphic to $C\left(S^{*} Y\right) \otimes \mathcal{K}$, where $S^{*} Y$ is the sphere bundle in $T^{*} Y$.

Proof. First of all notice that $C$ is isomorphic to $C_{0}\left(T^{*} Y \backslash Y\right) \rtimes \mathbb{R}_{+}^{*}$ : Indeed, the exact sequence

$$
0 \rightarrow C_{0}\left(T^{*} Y \backslash Y\right) \rightarrow C_{0}\left(T^{*} Y\right) \rightarrow C(Y) \rightarrow 0
$$

induces the exact sequence

$0 \rightarrow C_{0}\left(T^{*} Y \backslash Y\right) \rtimes \mathbb{R}_{+}^{*} \rightarrow C_{0}\left(T^{*} Y\right) \rtimes \mathbb{R}_{+}^{*} \rightarrow C(Y) \rtimes \mathbb{R}_{+}^{*}=C_{0}\left(Y \times \mathbb{R}_{+}^{*}\right) \rightarrow 0$.

But $T^{*} Y \backslash Y \simeq S^{*} Y \times \mathbb{R}_{+}^{*}$, so that $C_{0}\left(T^{*} Y \backslash Y\right) \simeq C\left(S^{*} Y\right) \otimes C_{0}\left(\mathbb{R}_{+}^{*}\right)$ and

$$
C_{0}\left(T^{*} Y \backslash Y\right) \rtimes \mathbb{R}_{+}^{*} \simeq C\left(S^{*} Y\right) \otimes C_{0}\left(\mathbb{R}_{+}^{*}\right) \rtimes \mathbb{R}_{+}^{*} .
$$

Now

$$
C_{0}\left(\mathbb{R}_{+}^{*}\right) \rtimes \mathbb{R}_{+}^{*} \simeq C^{*}\left(\mathbb{R}_{+}^{*}\right) \rtimes \mathbb{R}_{+}^{*} \simeq C^{*}\left(\mathbb{R}_{+}^{*} \rtimes \mathbb{R}_{+}^{*}\right) \simeq C^{*}\left(\mathbb{R}_{+}^{*} \times \mathbb{R}_{+}^{*}\right) \simeq \mathcal{K},
$$

where we again used Renault's result for the second isomorphism and the isomorphism of $\mathbb{R}_{+}^{*} \rtimes \mathbb{R}_{+}^{*}$ with the pair groupoid $\mathbb{R}_{+}^{*} \times \mathbb{R}_{+}^{*}$ for the third. This ends the proof.

\section{Identification of the ideal with the singular Green operators}

We have just shown that $\mathcal{I}$ is an extension of $C_{0}\left(S^{*} Y\right) \otimes \mathcal{K}$ by $\mathcal{K}$, and this is also the case for the algebra of singular Green operators. The main result is the following:

Theorem 2. The $C^{*}$-algebra $\mathcal{I}$ is isomorphic to $\Psi \otimes \mathcal{K}$.

Proof. We shall prove that the extensions

$$
0 \rightarrow \mathcal{K} \rightarrow \mathcal{I} \rightarrow C\left(S^{*} Y\right) \otimes \mathcal{K} \rightarrow 0
$$

and

$$
0 \rightarrow \mathcal{K} \rightarrow \Psi \otimes \mathcal{K} \rightarrow C\left(S^{*} Y\right) \otimes \mathcal{K} \rightarrow 0
$$

satisfy the conditions of a theorem by Voiculescu which we recall now.

D. Voiculescu proved in [31] (look also at the survey [30], Theorem 10.9) that if two extensions $0 \rightarrow \mathcal{K} \rightarrow D_{1} \rightarrow A \rightarrow 0$ and $0 \rightarrow \mathcal{K} \rightarrow D_{2} \rightarrow A \rightarrow 0$ are such that 
- $D_{1}$ and $D_{2}$ define the same element in $\operatorname{Ext}(A)$,

- $D_{1}$ and $D_{2}$ are not unital,

- $\mathcal{K}$ is essential in $D_{1}$ and in $D_{2}$,

then $D_{1}$ and $D_{2}$ are isomorphic. Recall that an ideal $J$ of $D$ is essential if and only if for every $x \in D$ : if $x \neq 0$ then there exists $y \in J$ such that $x y \neq 0$.

Let us apply this important result in our context.

First of all, $\mathcal{I}$ and $\Psi \otimes \mathcal{K}$ are non-unital since their quotients by the compacts are isomorphic to $C\left(S^{*} Y\right) \otimes \mathcal{K}$, which is non-unital. Since the algebra of compact operators on a Hilbert space $\mathscr{H}$ is essential in any $\mathrm{C}^{*}$-algebra included in $\mathscr{L}(\mathscr{H})$, we obtain that $\mathcal{K}_{X}$ is essential in $\Psi \otimes \mathcal{K}_{\mathbb{R}_{+}}$. For the other algebra, notice that $C^{*}\left(Y \times Y \times \mathbb{R}_{+}^{*}\right)$ is an essential ideal of $C^{*}\left({ }^{\text {ad }}(Y \times Y)\right)$, thus its crossed product by $\mathbb{R}_{+}^{*}$ is also an essential ideal of $C^{*}\left({ }^{\text {ad }}(Y \times Y)\right) \rtimes \mathbb{R}_{+}^{*}$, hence of $\mathcal{I}$ (see [16]).

Now it remains to show that the extensions give rise to the same element of $\operatorname{Ext}\left(C\left(S^{*} Y\right) \otimes \mathcal{K}\right)$. But since $C\left(S^{*} Y\right) \otimes \mathcal{K}$ is separable, $\mathrm{KK}_{1}\left(C\left(S^{*} Y\right) \otimes \mathcal{K}, \mathbb{C}\right)$ is isomorphic to the group of invertibles of $\operatorname{Ext}\left(C\left(S^{*} Y\right) \otimes \mathcal{K}\right)$ due to a result of Kasparov ([14]). The $\mathrm{C}^{*}$-algebra $C\left(S^{*} Y\right) \otimes \mathcal{K}$ being nuclear, $\operatorname{Ext}\left(C\left(S^{*} Y\right) \otimes \mathcal{K}\right)$ is actually a group, thus it is isomorphic to $\mathrm{KK}_{1}\left(C\left(S^{*} Y\right) \otimes \mathcal{K}, \mathbb{C}\right)$.

The element $i_{S} \in \mathrm{KK}_{1}\left(C\left(S^{*} Y\right) \otimes \mathcal{K}, \mathbb{C}\right) \simeq \operatorname{Ext}\left(C\left(S^{*} Y\right) \otimes \mathcal{K}\right)$ associated to the extension

$$
0 \rightarrow \mathcal{K}_{X} \rightarrow \Psi \otimes \mathcal{K}_{\mathbb{R}_{+}} \rightarrow C\left(S^{*} Y\right) \otimes \mathcal{K}_{\mathbb{R}_{+}} \rightarrow 0
$$

provides a map $K_{1}\left(C\left(S^{*} Y\right) \otimes \mathcal{K}_{\mathbb{R}_{+}}\right) \simeq K_{1}\left(C\left(S^{*} Y\right)\right) \rightarrow K_{0}(\mathbb{C})=\mathbb{Z}$, which is the analytic index.

For the class of the extension

$$
0 \rightarrow \mathcal{K} \rightarrow \mathcal{I} \rightarrow C\left(S^{*} Y\right) \otimes \mathcal{K} \rightarrow 0,
$$

let us consider first the extension

$$
0 \rightarrow C^{*}\left(Y \times Y \times \mathbb{R}_{+}^{*}\right) \rightarrow C^{*}\left({ }^{\mathrm{ad}}(Y \times Y)\right) \rightarrow C_{0}\left(T^{*} Y\right) \rightarrow 0,
$$

whose class is denoted by $i_{T} \in \mathrm{KK}_{1}\left(C_{0}\left(T^{*} Y\right), \mathcal{K} \otimes C_{0}\left(\mathbb{R}_{+}^{*}\right)\right) \simeq \mathrm{KK}_{0}\left(C_{0}\left(T^{*} Y\right), \mathcal{K}\right)$.

It induces the extension

$$
\begin{aligned}
0 \rightarrow C^{*}\left(Y \times Y \times \mathbb{R}_{+}^{*}\right) \rtimes \mathbb{R}_{+}^{*} & \simeq \mathcal{K} \rightarrow C^{*}\left({ }^{\mathrm{ad}}(Y \times Y)\right) \rtimes \mathbb{R}_{+}^{*} \\
& \simeq C^{*}(G) \rightarrow C_{0}\left(T^{*} Y\right) \rtimes \mathbb{R}_{+}^{*} \rightarrow 0
\end{aligned}
$$

whose class is denoted by $\alpha \in \mathrm{KK}_{1}\left(C_{0}\left(T^{*} Y\right) \rtimes \mathbb{R}_{+}^{*}, \mathcal{K}\right)$.

The relation between $i_{T}$ and $i_{S}$ is made clear by considering the exact sequence

$$
0 \rightarrow C_{0}\left(T^{*} Y\right) \rightarrow C\left(B^{*} Y\right) \rightarrow C\left(S^{*} Y\right) \rightarrow 0,
$$

where $B^{*} Y$ is the ball bundle over $Y$. By $\psi \in \mathrm{KK}_{1}\left(C\left(S^{*} Y\right), C_{0}\left(T^{*} Y\right)\right)$ we denote its class, and one has the well-known equality

$$
i_{S}=\psi i_{T} .
$$


For the convenience of the reader, we now recall the diagram (14):

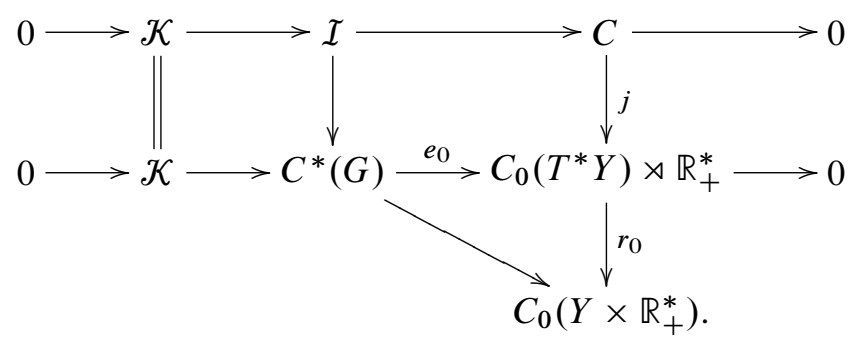

Let us denote the class of the first sequence by $\partial \in \mathrm{KK}_{1}(C, \mathcal{K})$; it is thus given by the Kasparov product

$$
\partial=j_{*} \alpha .
$$

Let us make the relation between $j_{*}$ and $\varphi$ precise.

Consider the following commutative diagram:

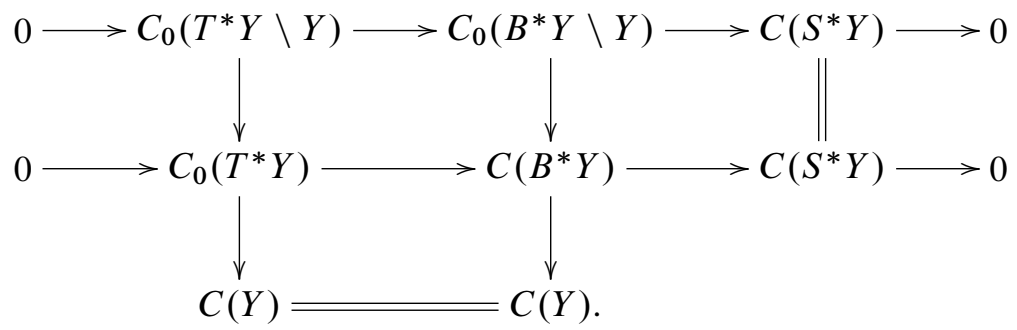

The first exact sequence actually decomposes as

$$
0 \rightarrow C\left(S^{*} Y\right) \otimes C_{0}\left(\mathbb{R}_{+}^{*}\right) \rightarrow C\left(S^{*} Y\right) \otimes C_{0}\left(\mathbb{R}_{+}^{*} \cup\{\infty\}\right) \rightarrow C\left(S^{*} Y\right) \rightarrow 0
$$

so that its $\mathrm{KK}_{1}$-class is the identity of $\mathrm{KK}_{1}\left(C\left(S^{*} Y\right), C\left(S^{*} Y\right) \otimes C_{0}\left(\mathbb{R}_{+}^{*}\right)\right)$.

There is an action of $\mathbb{R}_{+}^{*}$ on each algebra of the previous diagram, which is trivial on $C\left(S^{*} Y\right)$ and $C(Y)$. This gives the following:

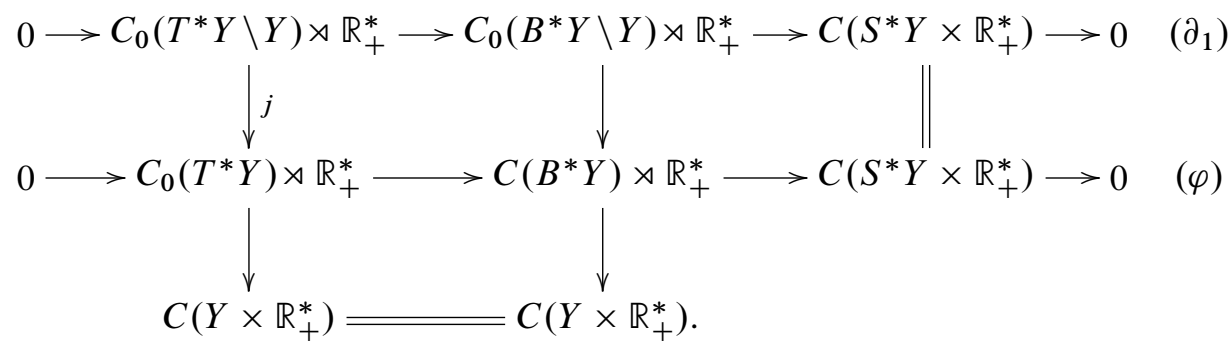

Denote by $\partial_{1}$ (resp. $\varphi$ ) the class of the first (resp. second) exact sequence of this diagram, and by $j_{*} \in \mathrm{KK}\left(C_{0}\left(T^{*} Y \backslash Y\right), C_{0}\left(T^{*} Y\right)\right)$ the element induced by $C_{0}\left(T^{*} Y \backslash Y\right) \rightarrow C_{0}\left(T^{*} Y\right)$. One has thus the equality

$$
\varphi=\partial_{1} j_{*}
$$


so that

$$
\partial=j_{*} \alpha=\partial_{1}^{-1} \varphi \alpha .
$$

But $\varphi \alpha$ is the image of $\psi i_{T}$ under the Thom-Connes isomorphism, and $\partial_{1}$ is also a Thom-Connes element in KK-theory. Hence the classes in $\mathrm{KK}_{1}$ of the extensions of $\mathcal{I}$ and of $\Psi \otimes \mathcal{K}$ are the same. Voiculescu's theorem implies that these algebras are isomorphic.

Corollary 1. The algebra of singular Green operators is isomorphic to I, an ideal of $C^{*}\left({ }^{\mathrm{ad}}(Y \times Y) \rtimes \mathbb{R}_{+}^{*}\right)$.

Proof. This is a direct consequence of Theorem 2, since the algebra of singular Green operators is isomorphic to $\Psi \otimes \mathcal{K}$.

\section{References}

[1] J. Aastrup, R. Nest, and E. Schrohe, A continuous field of $C^{*}$-algebras and the tangent groupoid for manifolds with boundary. J. Funct. Anal. 237 (2006), 482-506.

Zbl 1131.58017 MR 2230347

[2] M. F. Atiyah and I. M. Singer, The index of elliptic operators: I. Ann. of Math. (2) 87 (1968), 484-530. Zbl MR 0236950

[3] L. Boutet de Monvel, Comportement d'un opérateur pseudo-différentiel sur une variété à bord. I. La propriété de transmission. J. Analyse Math. 17 (1966), 241-253.Zbl 0161.0790 MR 0239254

[4] L. Boutet de Monvel, Comportement d'un opérateur pseudo-différentiel sur une variété à bord. II. Pseudo-noyaux de Poisson. J. Analyse Math. 17 (1966), 255-304. Zbl 0161.0790 MR 0239255

[5] L. Boutet de Monvel, Boundary problems for pseudo-differential operators. Acta Math. 126 (1971), 11-51. Zbl 0206.39401 MR 0407904

[6] L. G. Brown, Stable isomorphism of hereditary subalgebras of $C^{*}$-algebras. Pacific $J$. Math. 71 (1977), 335-348. Zbl 0362.46042 MR 0454645

[7] L. G. Brown, P. Green, and M. A. Rieffel, Stable isomorphism and strong Morita equivalence of $C^{*}$-algebras. Pacific J. Math. 71 (1977), 349-363. Zbl 0362.46043 MR 0463928

[8] A. Connes, Noncommutative geometry. Academic Press, San Diego, CA, 1994. Zbl 0818.46076 MR 1303779

[9] B. V. Fedosov, Index theorems. In Partial differential equations VIII, Encyclopaedia Math. Sci. 65, Springer, Berlin 1996, 155-251. Zbl 0884.58087 MR 1135117

[10] B. V. Fedosov, F. Golse, E. Leichtnam, and E. Schrohe, The noncommutative residue for manifolds with boundary. J. Funct. Anal. 142 (1996), 1-31. Zbl 0877.58005 MR 1419415

[11] G. Grubb, Functional calculus of pseudodifferential boundary problems. 2nd ed., Progr. Math. 65, Birkhäuser, Boston 1996. Zbl 0844.35002 MR 1385196 
[12] G. Grubb and E. Schrohe, Trace expansions and the noncommutative residue for manifolds with boundary. J. Reine Angew. Math. 536 (2001), 167-207. Zbl 0980.58017 MR 1837429

[13] L. Hörmander, Pseudo-differential operators and hypoelliptic equations. In Singular integrals (Chicago, Ill., 1966), Proc. Sympos. Pure Math. X, Amer. Math. Soc., Providence, R.I., 1967, 138-183. Zbl 0167.09603 MR 0383152

[14] G. G. Kasparov, The operator $K$-functor and extensions of $C^{*}$-algebras. Izv. Akad. Nauk SSSR Ser. Mat. 44 (1980), 571-636; English transl. Math.-USSR Izv. 16 (1981), 513-572. Zbl 0464.46054 MR 582160

[15] J. J. Kohn and L. Nirenberg, An algebra of pseudo-differential operators. Comm. Pure Appl. Math. 18 (1965), 269-305. Zbl 0171.35101 MR 0176362

[16] M. Kusuda, Characterizations of certain classes of hereditary $C^{*}$-subalgebras. Proc. Amer. Math. Soc. 116 (1992), 999-1005. Zbl 0809.46077 MR 1127142

[17] R. Lauter, B. Monthubert, and V. Nistor, Pseudodifferential analysis on continuous family groupoids. Doc. Math. 5 (2000), 625-655. Zbl 0961.22005 MR 1800315

[18] R. Lauter, B. Monthubert, and V. Nistor, Spectral invariance for certain algebras of pseudodifferential operators. J. Inst. Math. Jussieu 4 (2005), 405-442. Zbl 1088.35087 MR 2197064

[19] S. T. Melo, R. Nest, and E. Schrohe, $C^{*}$-structure and $K$-theory of Boutet de Monvel's algebra. J. Reine Angew. Math. 561 (2003), 145-175. Zbl 1043.19003 MR 1998610

[20] S. T. Melo, T. Schick, and E. Schrohe, A $K$-theoretic proof of Boutet de Monvel's index theorem for boundary value problems. J. Reine Angew. Math. 599 (2006), 217-233. Zbl 1121.19001 MR 2279103

[21] B. Monthubert, Groupoids and pseudodifferential calculus on manifolds with corners. $J$. Funct. Anal. 199 (2003), 243-286. Zbl 1025.58009 MR 1966830

[22] B. Monthubert and F. Pierrot, Indice analytique et groupoïdes de Lie. C. R. Acad. Sci. Paris Sér. I Math. 325 (1997), 193-198. Zbl 0955.22004 MR 1467076

[23] R. Nest and E. Schrohe, Dixmier's trace for boundary value problems. Manuscripta Math. 96 (1998), 203-218. Zbl 0914.35158 MR 1624529

[24] V. Nistor, A. Weinstein, and P. Xu, Pseudodifferential operators on differential groupoids. Pacific J. Math. 189 (1999), 117-152. Zbl 0940.58014 MR 1687747

[25] S. Rempel and B.-W. Schulze, Index theory of elliptic boundary problems. Math. Lehrbücher Monogr., II. Abt.: Math. Monogr. 55, Akademie-Verlag, Berlin 1982. Zbl 0504.35002 MR 0690065

[26] J. Renault, A groupoid approach to $C^{*}$-algebras. Lecture Notes in Math. 793, Springer, Berlin 1980. Zbl 0433.46049 MR 0584266

[27] M. Rørdam, Stable $C^{*}$-algebras. In Operator algebras and applications, Adv. Stud. Pure Math. 38, Math. Soc. Japan, Tokyo 2004, 177-199. Zbl 1065.46037 MR 2059808

[28] E. Schrohe, Fréchet algebra techniques for boundary value problems on noncompact manifolds: Fredholm criteria and functional calculus via spectral invariance. Math. Nachr. 199 (1999), 145-185. Zbl 0923.58054 MR 1676318 
[29] E. Schrohe, A short introduction to Boutet de Monvel's calculus. In Approaches to singular analysis (Berlin, 1999), Oper. Theory Adv. Appl. 125, Birkhäuser, Basel 2001, 85-116. Zbl 0985.35122 MR 1827171

[30] G. Skandalis, Kasparov's bivariant $K$-theory and applications. Exposition. Math. 9 (1991), 193-250. Zbl 0746.19008 MR 1121156

[31] D. Voiculescu, A non-commutative Weyl-von Neumann theorem. Rev. Roumaine Math. Pures Appl. 21 (1976), 97-113. Zbl 0335.46039 MR 0415338

Received April 12, 2006

J. Aastrup, Institut für Analysis, Leibniz Universität Hannover, Welfengarten 1, 30167 Hannover, Germany.

Present address: Mathematisches Institut, Georg-August-Universität Göttingen, Bunsenstraße 3-5, 37073 Göttingen, Germany

E-mail: aastrup@math.uni-hannover.de; aastrup@uni-math.gwdg.de

S. T. Melo, Instituto de Matemática e Estatística, Universidade de São Paulo, Rua do Matão 1010, São Paulo SP, 05508-090, Brazil

E-mail: toscano@ime.usp.br

B. Monthubert, Institut de Mathématiques de Toulouse, Université Paul Sabatier, 118 route de Narbonne, 31062 Toulouse Cedex 9, France

E-mail: bertrand@monthubert.net

E. Schrohe, Institut für Analysis, Leibniz Universität Hannover, Welfengarten 1, 30167 Hannover, Germany

E-mail: schrohe@math.uni-hannover.de 